\title{
No need for a social cue! A masked magician can also trick the audience in the vanishing ball illusion
}

\author{
Cyril Thomas $^{1} \cdot$ André Didierjean $^{1}$
}

Published online: 16 December 2015

(C) The Psychonomic Society, Inc. 2015

\begin{abstract}
In the vanishing ball illusion (VBI), a magician throws a ball up in the air twice, after which he pretends to toss it up again, when in fact it remains secretly concealed in his hand. Observers perceive an imaginary ball disappearing into the air. According to Kuhn and Land (2006), the VBI during the fake throw is mediated by the magician's gaze and/or head direction (also called "social cues") as he looks toward the imaginary ball. The aim of this article is to test an alternative interpretation. According to our hypothesis, the magician's social cues are not essential to the VBI. We compared the numbers of participants experiencing the VBI when the magician's social cues were directed toward the illusory ball and when the magician's social cues were either hidden behind a black mask (Exp. 1) or stationary (Exp. 2). The results showed that the number of observers experiencing the VBI was high (almost two-thirds of the participants), regardless of whether the magician's social cueing was directed toward the illusion, hidden behind a mask, or stationary. In a third experiment (Exp. 3), we replicated Kuhn and Land's initial results and attempted to further explain their "anti-illusion" social-cue effect. This study confirms that social cueing is not required in the VBI: Its presence did not increase the number of participants experiencing the illusion.
\end{abstract}

Keywords Magic $\cdot$ Social cueing $\cdot$ Perception $\cdot$ Illusion

Cyril Thomas

cyril.thomas@univ-fcomte.fr

1 University of Franche-Comté, 30 rue Mégevand, 25030 Besançon, France
Imagine a magician playing with a small red ball. He throws it into the air once, twice, and suddenly, during the third throw, the ball rises and disappears magically into thin air. This old illusion is known as "the vanishing ball illusion" (VBI). The first study about this illusion dates back to the beginning of the 20th century (Triplett, 1900). Triplett demonstrated the VBI in live conditions: "The operator sitting behind the teacher's desk threw the ball about three feet in the air, catching it and letting the hand sink low behind the table. The second throw was four or five feet in height. On its return it was dropped between the legs but the hands went up with the regular throwing movement and were held as if awaiting the descent of the ball" (Triplett, 1900, p. 492). The results of this study indicated that almost half of the participants ( $47 \%$ ) were sensitive to the illusion and "saw" the ball go up and disappear. According to Triplett, the repetition of the two real throws creates expectations in the spectators' minds, and these expectations enhance the illusion during the last, fake throw. A century after Triplett's study, Kuhn and Land (2006) reexplored the VBI with modern experimental tools. In their study, they showed spectators a video version of the VBI. In this version, during the last, fake throw, instead of lapping the ball (see Triplett, 1900), the magician secretly kept the ball hidden in his right hand (what magicians call "palming the ball").

One of the principal aims of Kuhn and Land's (2006) study was to track participants' eye movements during the VBI in order to investigate the impact of the magician's social cues on illusion sensitivity. The social cue here was the magician's gaze (and/or head direction), which relies on our tendency to look at and focus attention where the speaker is looking (e.g., Driver et al., 1999; Mansfield, Farroni, \& Johnson, 2003). Many studies have shown that this social cue can be used by magicians to misdirect spectators' attention (for the importance of social cues in misdirection, see Kuhn, Tatler, \& Cole, 2009; Kuhn, Teszka, 
Tenaw, \& Kingstone, 2015; Tachibana, 2014; Tatler \& Kuhn, 2007), but few authors have studied the role of social cueing in perceptual illusions. To investigate this question, Kuhn and Land proposed showing two versions of the VBI video to participants. In the "social cues pro-illusion" condition, the magician watched an imaginary ball moving upward during the fake throw. In the "social cues anti-illusion" condition, the magician looked at his right hand (the hand concealing the ball) during the fake throw.

The results showed that in the pro-illusion condition, almost two-thirds of the observers (68\%) actually believed they saw the ball vanish into the air, significantly more than in the anti-illusion condition (32\%). By tracking participants' eye movements, Kuhn and Land (2006) showed that most of the participants tended to look at the magician's face before looking at the ball, suggesting that the visual system uses information about where the magician is looking as a way of predicting the ball's location. According to the authors, these results show that the illusion is mediated by social cueing. In other words, the magician's social cues increased the number of participants experiencing the VBI.

The aim of the present study was to propose and test an alternative interpretation of Kuhn and Land's (2006) results. We hypothesized that social cueing by the magician is not needed to find a high number of participants experiencing the VBI. The fact that Kuhn and Land showed that the antiillusion condition could substantially lower the number of participants who were sensitive to the illusion does not prove that the magician's social cueing in the pro-illusion condition increased the number of participants experiencing the illusion. In the anti-illusion condition, Kuhn and Land did not control the presence versus absence of a social cue, but the impact of the presence of a social cue toward another area of the scene. This difference could be essential to interpreting Kuhn and Land's results.

In the anti-illusion condition, the magician's gaze and head position directed the spectators' attention to his suspicious hand (his right hand, secretly palming the ball), orienting the spectators' minds toward the solution of the trick: The ball was secretly palmed. This would make the unnatural, tense position of the magician's hand during the fake throw more salient for these participants. According to our alternative hypothesis, fewer participants experienced the VBI in the antiillusion condition because the magician's social cues attracted the participants' attention to the solution of the trick, not because the magician did not follow the ball with his gaze/head.

Similarly, if participants tended to look at the magician's face before each throw in the pro-illusion condition, this does not constitute proof that the magician's social cueing enhanced the number of participants sensitive to the VBI. In a recent article, Cui, Otero-Millan, Macknik, King, and Martinez-Conde (2011) showed that social cueing failed to improve a misdirection act, and that participants naturally followed a real or a fake transfer of a coin from one hand to the other, regardless of whether the magician's social cueing was visible or occluded behind a black mask. Similarly, even if a magician's social cueing is occluded during the VBI, participants might still look up during the fake throw because they expect the ball to be thrown upward. A large body of research on "representational momentum" (Freyd \& Finke, 1984; for reviews, see Didierjean, Ferrari, \& Blättler, 2014; Hubbard, 2005, 2015) has also shown that without any social cueing, observers have a natural tendency to see the final stopping point of a moving object as displaced forward in the object's direction of motion.

The purpose of the present study was to find out whether the presence of the magician's social cues really increases the number of participants experiencing the VBI. This question is important because, if social cueing is not the key process in the VBI, then the nature of the anticipation processes behind this magic trick are still currently unknown (e.g., Rensink \& Kuhn, 2015; Thomas, Didierjean, Maquestiaux, \& Gygax, 2015).

\section{Experiment 1}

The aim of Experiment 1 was to find out whether the VBI is mediated by the magician's social cues, by comparing the numbers of participants experiencing the VBI when the magician's social cueing is visible and when these cues are hidden behind a mask.

\section{Method}

Participants A total of 62 students ( 50 female, 12 male; mean age $=20$ years, $S D=1.8$ ) from the University of FrancheComté, France, participated in the experiment. All participants volunteered for the experiment and reported normal or corrected-to-normal vision. They thought they were participating in an experiment about memory and did not know they would see a magic trick.

Apparatus and stimuli The stimuli were displayed on a Dell Latitude E5500 computer (monitor diagonal size $=15.4$ in., resolution $=1,280 \times 800$ pixels). The viewing distance was approximately $60 \mathrm{~cm}$. Two versions of the same video were created for the experiment (see Appendix B for video links). The first version, called "social-cue," was similar to the version used by Kuhn and Land (2006); see Fig. 1.

The second version, called "no-social-cue," was similar to the social-cue version, except that the magician's head and gaze were hidden behind a black mask (see Fig. 2) throughout the video, preventing the magician's social cues from influencing the observers. 


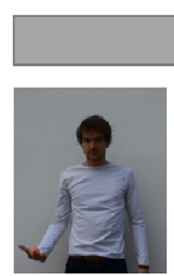

a
First real throw

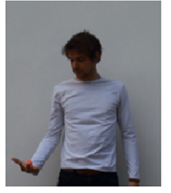

b

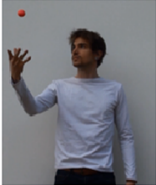

C

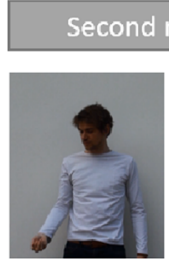

d

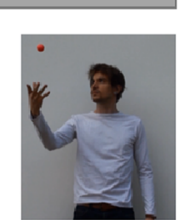

e

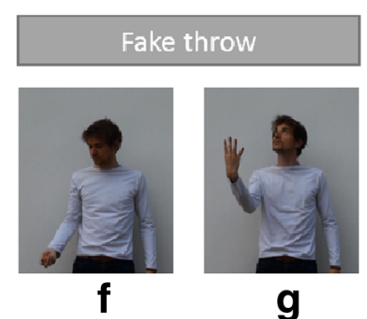

Fig. 1 Description of the social-cue condition. a The magician looks at the participant. b He looks at the ball held in his right hand. $\mathbf{c}$ He throws the ball up in the air and follows it with his head and gaze. $\mathbf{d}$ The magician catches the ball. e He throws the ball up in the air a second time and follows the ball with his head and gaze. $\mathbf{f}$ The ball is caught for the second time. $g$ The magician pretends to throw the ball up in the air (while secretly concealing it in the palm of his right hand), and his eyes and head follow the imaginary ball moving upward
Procedure Half of the participants watched the social-cue version of the video, and the other half watched the nosocial-cue version. ${ }^{1}$ Participants watched the video individually and only once. They were asked to watch the video carefully, and immediately after, they had to use the mouse to click on the exact location where they "saw" the ball for the last time. Then they answered a French adaptation of Kuhn and Land's (2006) ball illusion questionnaire (see Appendix A). The purpose of the questionnaire was to find out whether or not the participant had experienced the VBI. Participants were considered "sensitive" to the VBI when they both located the ball during the last throw above the fingers of the magician's hand (Question 1 of the ball illusion questionnaire) and verbally reported that they saw the ball moving up during the last throw (Questions 2, 4, and 5). Participants were considered "not sensitive" to the VBI when they located the ball during the last throw on or below the magician's hand/fingers (Question 1) or when they verbally reported that they did not see the ball moving up or going off the screen during the last throw (Questions 2, 4, and 5). Question 3 was a qualitative question that indicated whether participants sensitive to the illusion were able to, a posteriori, find the secret of the trick.

\section{Results and discussion}

Our goal here was to determine whether the magician's social cueing is essential for experiencing the VBI. We conducted a chi-square test to compare the percentages of participants experiencing the VBI in each group (social-cue vs. no-socialcue condition). The results showed that the percentage of participants experiencing the VBI in the social-cue condition $(67.74 \%)$ was not significantly different from the percentage in the no-social-cue condition $(70.97 \%), \chi^{2}(1, N=62)=0.29$, $p=.59$. The results also showed that the percentage of participants who experienced the VBI and "saw" the ball go off the screen during the final throw in the social-cue condition ( $80 \%)$

\footnotetext{
${ }^{1}$ Six males and 25 females for the social-cue condition, eight males and 23 females for the no-social-cue condition.
}

was not significantly different from the percentage in the nosocial-cue condition $(72.72 \%), \chi^{2}(1, N=62)=0.30, p=.58$. See Fig. 3 for details about the location distributions in each condition (social-cue condition and no-social-cue condition). As in the Kuhn and Land (2006) results, the answers to Question 3 of the ball illusion questionnaire ("How do you think the illusion was created?") showed that participants who were sensitive to the illusion typically claimed that it was created by an accomplice catching the ball beyond the top of the screen or by video editing (for participants who saw the ball disappear while it was still on the screen). Participants who were not sensitive to the illusion typically claimed that the magician kept the ball hidden behind his hand, in his sleeve, or that he let it drop below the screen. ${ }^{2}$

These results are consistent with our hypothesis. They do not support Kuhn and Land's (2006) hypothesis that the VBI is mediated by the magician's gaze or head position.

However, according to the amodal-completion theory (Kanizsa \& Gerbino, 1982; Michotte, Thines, \& Crabbe, 1991), when a part of an object or volume is occluded behind another object, the perceptual system automatically fills in the missing information in order to obtain a complete impression of the total object, on the basis of probabilistic physical laws or expectations (Tse, 1999; van Lier \& Wagemans, 1999). Moreover, Hegdé, Fang, Murray, and Kersten (2008) used functional magnetic resonance imaging to show that the amodal completion of occluded objects activates the same cortical areas (lateral occipital complex and dorsal objectselective areas) as the perception of the whole object. Thus, even when the magician's social cueing was hidden behind a black mask in the no-social-cue condition, the participants may have completed the occluded zone on the basis of their expectations, thereby "perceiving" the magician's social cueing as if it were not occluded (as in the social-cue condition). The aim of the next experiment was to confirm that the magician's social cueing is not essential for experiencing the VBI, using a

\footnotetext{
${ }^{2}$ None of the participant in the no-social-cue condition saw the ball disappear behind the black mask (or claimed that it had) during the last throw.
} 


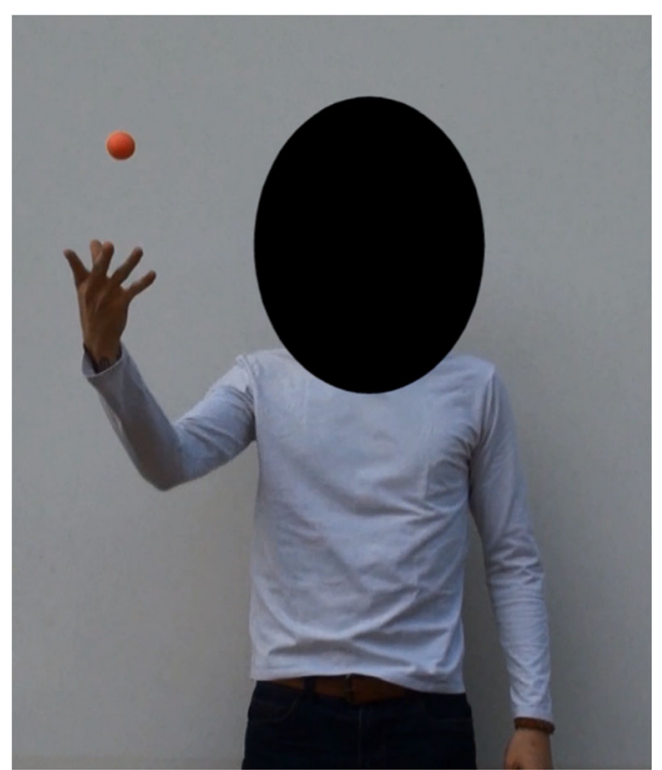

Fig. 2 Illustration of the black mask used for the no-social-cue condition

new no-social-cue condition that made any potential amodal completion impossible.

\section{Experiment 2}

The aim of Experiment 2 was to find out whether the VBI is mediated by the magician's social cues, by comparing the numbers of participants experiencing the VBI when the magician's gaze and head followed both the real ball (first two throws) and the imaginary ball (last, "fake" throw) and when his gaze and head position were static (looking at the camera) during the entire trick.

\section{Method}

Participants A total of 70 students (48 female, 22 male $^{3}$; mean age $=21$ years, $S D=3.2$ ) from the University of Franche-Comte, France, participated in the experiment. All participants volunteered and reported normal or corrected-tonormal vision. They thought they were participating in an experiment about memory and did not know they would see a magic trick.

Apparatus and stimuli The materials were similar to those used in Experiment 1, except that in the second version of

${ }^{3}$ Eight males and 22 females for the social-cue condition, 14 males and 26 females for the no-social-cue condition. the video, again called "no-social-cue," the magician's head and gaze were visible (looking at the camera) and stationary throughout the video, instead of being hidden behind a mask (see Appendix B for video links). This prevented the magician's social cues from influencing the observers; see Fig. 4. Moreover, to control the light conditions and to have the same background in the two versions of the video, the filming conditions were made as similar as possible. ${ }^{4}$

\section{Results and discussion}

Our goal here was to confirm that the magician's social cueing is not essential for experiencing the VBI. We conducted a chi-square test to compare the percentages of participants experiencing the VBI in each group (socialcue vs. no-social-cue condition). The results showed that the percentage of participants experiencing the VBI in the social-cue condition $(73.33 \%)$ was not significantly different from the percentage in the no-social-cue condition $(87.5 \%), \chi^{2}(1, N=70)=2.27, p=.13$.

The results also showed that the percentage of participants experiencing the VBI and "seeing" the ball go outside the frame of the screen during the final throw in the social-cue condition $(77.27 \%)$ was not significantly different from the percentage in the no-social-cue condition $(91.43 \%)$, $\chi^{2}(1, N=70)=2.24, p=.13$. See Fig. 5 for details about the location distributions for each condition (socialcue and no-social-cue). The answers to Question 3 of the ball illusion questionnaire ("How do you think the illusion was created?") were similar to those obtained in Experiment 1.

These results confirm our previous ones and do not support Kuhn and Land's (2006) hypothesis that the VBI is mediated by the magician's gaze or head position. Our results are still somewhat surprising, knowing the impact of the magician's social cues on other kinds of tricks, such as misdirection tricks (e.g., Kuhn, Caffaratti, Teszka, \& Rensink, 2014; Kuhn, Tatler, \& Cole, 2009; Kuhn et al., 2015). We assume that, unlike in misdirection acts, cues other than the magician's head or gaze direction (e.g., the two prior throws, the magician's hand motion during the last throw) in the VBI are strong enough to create a kind of "ceiling" effect on the number of participants sensitive to the illusion. These other cues may be sufficient to direct spectators' expectations and attention to the anticipated trajectory of the ball during the last, fake throw. According to several authors, these expectations may be key

\footnotetext{
${ }^{4}$ The two sequences were filmed at the same distance from the camera, with the same angle, background, and daylight. Moreover, the magician made the last throw as similar as possible to the others for each condition (same speed, same height of the hand at the end of the fake throw, same position of the "palming" hand).
} 

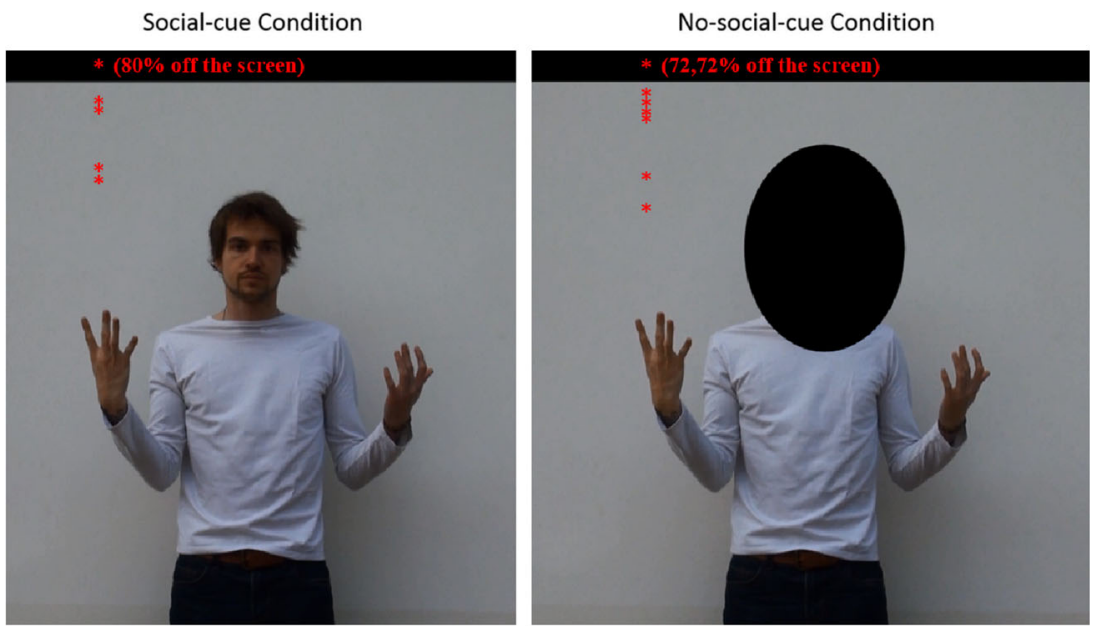

Fig. 3 Locations (heights) of the illusory ball during the last, fake throw as a function of the condition (social-cue condition on the left, no-social-cue condition on the right) for the 43 participants who experienced the vanishing ball illusion in Experiment 1

mechanisms in the VBI experience (Kuhn, Kourkoulou, \& Leekam's, 2010; Kuhn \& Land, 2006; Kuhn \& Rensink, 2015; Thomas et al., 2015; Triplett, 1900). However, even though the magician's social cues directed at the illusory ball fail to enhance the number of participants experiencing the VBI, these social cues would have the capacity to misdirect spectators' attention and reduce the VBI experience when the cues are directed toward the magician's hand (Kuhn \& Land, 2006). When the magician directs his gaze/head toward his hand, it is probable that he orients spectators' attention toward

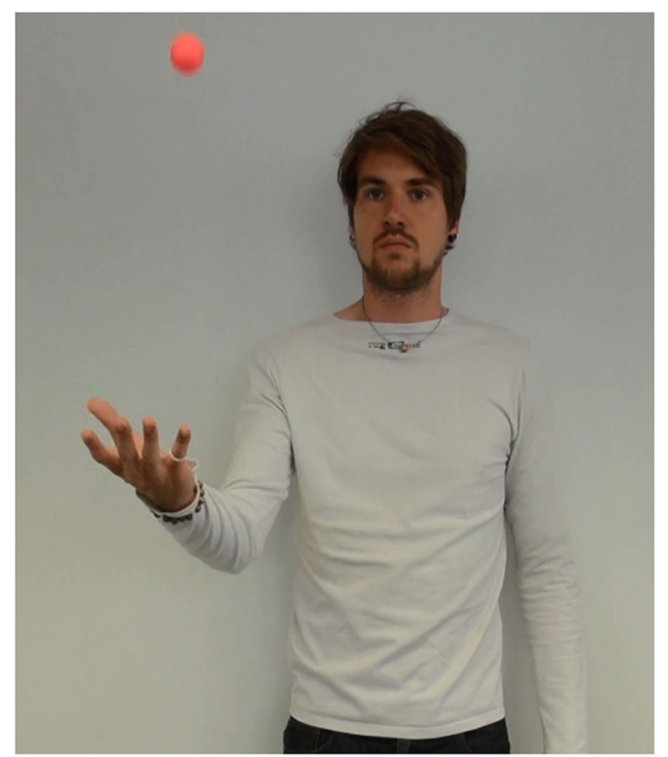

Fig. 4 Illustration of the stationary head position and gaze used for the no-social-cue condition. The procedure was the same as in Experiment 1 the solution of the trick (i.e., the ball is palmed) and misdirects attention from the illusory ball. As in misdirection acts, the illusion is broken when the magician actively "moves" his social cues from one place (the ball trajectory) to another (the magician's hand). That being said, one may ask what would happen if the magician's social cues were still fixed on the throwing hand throughout the video (during the two real throws and the fake one). In the "anti-illusion" condition (Kuhn \& Land, 2006), maybe the spectator's attention is more efficiently attracted by the "anti-illusion" social cues and oriented toward the solution of the trick (the ball is palmed) because the expected direction of the magician's gaze/head (toward the ball trajectory) changes to an unexpected direction (toward his hand). Indeed, according to Howard and Holcombe (2010), unexpected changes in the direction of a moving target (here, the magician's gaze/head direction) capture the attention.

The aim of Experiment 3 was to investigate whether the magician's social cues can reduce VBI sensitivity when they are fixed on the throwing hand either throughout the video or only during the last, fake throw (as in Kuhn \& Land, 2006).

\section{Experiment 3}

The aim of Experiment 3 was to find out whether VBI sensitivity can be reduced by the magician's social cues when they are directed toward his hand, by comparing the numbers of participants experiencing the VBI when the magician's gaze and head followed both the real ball (first two throws) and the imaginary ball (last, fake throw) and when his gaze and head position were fixed on the throwing 


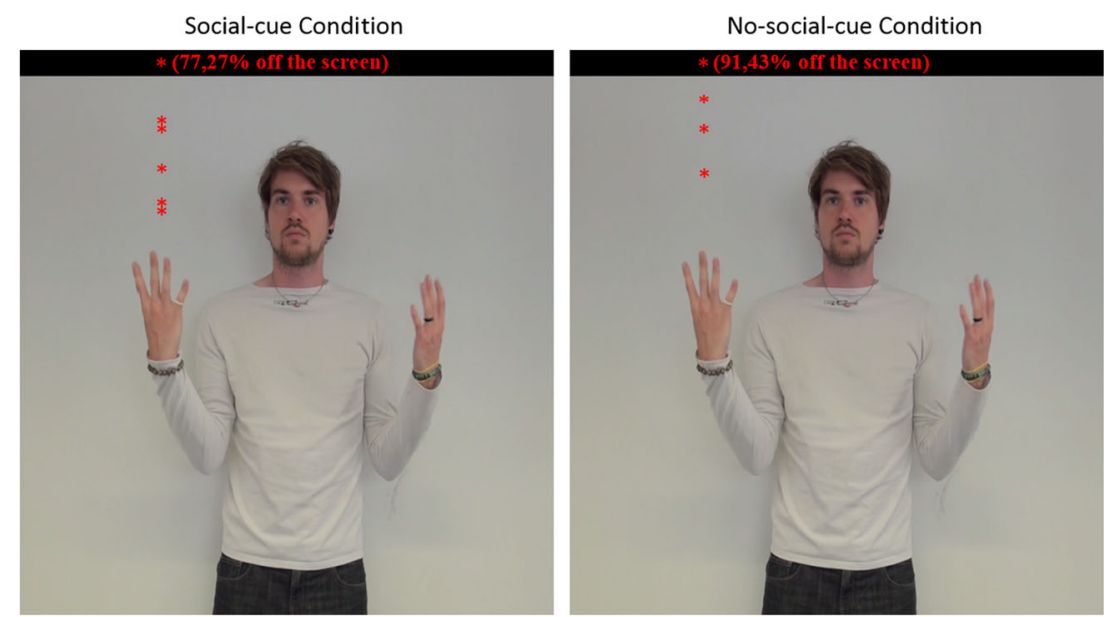

Fig. 5 Locations (heights) of the illusory ball during the last, fake throw as a function of the condition (social-cue condition on the left, no-social-cue condition on the right) for the 57 participants who experienced the vanishing ball illusion in Experiment 2

hand either throughout the video or only during the last, fake throw (the latter being a replication of Kuhn \& Land's, 2006, "anti-illusion" condition).

\section{Method}

Participants A total of 120 students (89 females, 31 males $^{5}$; mean age $=21$ years, $S D=2.4$ ) from the University of FrancheComté, France, participated in the experiment. All participants volunteered and reported normal or corrected-to-normal vision. They did not know they would see a magic trick.

Apparatus and stimuli The materials were similar to those used in Experiments 1 and 2. Three different video versions of the VBI were created for the experiment (see Appendix B for video links). The first version, called "social-cue," was similar to the version used by Kuhn and Land (2006). The second version, called "anti-illusion," was similar to the "antiillusion" version used by Kuhn and Land. In this version, the magician's gaze and head followed the real ball during the first two throws and were directed toward the throwing hand during the last, fake throw. In the third version, called "fixed-anti-illusion," the magician's gaze and head position were fixed on the throwing hand throughout the video. Moreover, to control the light conditions and to have the same background in the three versions of the video, the filming conditions were made as similar as possible.

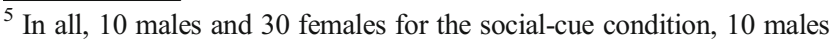
and 30 females for the anti-illusion condition, and 11 males and 29 females for the fixed-anti-illusion condition.
}

\section{Results and discussion}

Our goal here was to investigate whether the magician's social cues can reduce VBI sensitivity when they are fixed on the throwing hand throughout the video or only during the last, fake throw.

Our results showed that the percentage of participants experiencing the VBI in the social-cue condition (70 \%) was significantly higher than the percentage in the anti-illusion condition $(32.5 \%), \chi^{2}(1, N=80)=11.26, p<.001$, and higher than the percentage in the fixed-anti-illusion condition $(42.5 \%), \chi^{2}(1, N=80)=6.15, p<.05$. The percentage of participants experiencing the VBI in the anti-illusion condition $(32.5 \%)$ was not significantly different from the percentage in the fixed-anti-illusion condition (42.5\%), $\chi^{2}(1, N=80)=0.85, p=.36$.

The results of Experiment 3 replicated those reported by Kuhn and Land (2006): VBI sensitivity can be significantly reduced by the magician's social cues when they are directed toward his hand during the last, fake throw. Moreover, our results show that the reduction of VBI sensitivity is not significantly different, regardless of whether the magician's social cues are directed toward the suspicious hand only during the last, fake throw or throughout the video.

Taken together, the results of Experiment 3 did not indicate that the spectator's attention is more efficiently attracted toward the solution of the trick when the expected direction of the magician's gaze/head (toward the ball trajectory) changes to an unexpected direction (toward his hand). In "fixed-antiillusion" conditions, the magician's social cues may be strong enough to attract the attention of a ceiling number of the spectators toward the solution of the trick. However, we assume 
that in other tricks, such as misdirection tricks, an unexpected change in the direction of the magician's social cues might be more efficient than a "fixed-anti-illusion" social cue at misdirecting spectators' attention.

\section{General discussion}

The goal of the present study was to find out whether the VBI is mediated by the magician's social cues, by comparing the numbers of participants experiencing the VBI when the magician's social cues were visible and when those cues were hidden behind a mask (Exp. 1) or when the magician's head and gaze were stationary throughout the video (Exp. 2), preventing the magician's social cues from influencing the observers. In a third experiment (Exp. 3), we also replicated the initial results from Kuhn and Land (2006) and tested whether the magician's social cues could reduce VBI sensitivity when they were fixed on the throwing hand either throughout the video or only during the last, fake throw.

Overall, we hypothesized that social cues from the magician are not needed in order to observe a high number of participants experiencing the VBI. The results of both of the first experiments validated our hypothesis by showing that the number of participants experiencing the VBI in the no-socialcue condition was high (more than two-thirds of the participants) and was not significantly different from the percentage in the social-cue condition. However, Experiment 3 showed that, as was demonstrated by Kuhn and Land (2006), if the magician's gaze is fixed on the throwing hand (either throughout the video or during the last, fake throw), the number of participants experiencing the VBI decreased.

It is widely acknowledged that a magician's social cues can direct the attention of participants to a zone of interest (see Kuhn, Tatler, \& Cole, 2009; Kuhn et al., 2015; Tatler \& Kuhn, 2007), but on the basis of our results, social cueing is not required to create an effective VBI. Without any social cueing, the expectations of participants may be strong enough to bias their perception of the expected ball throw. Our results can also shed a different light on Kuhn, Kourkoulou, and Leekam's (2010) results. In their experiment, the authors showed that individuals with autism spectrum disorder (ASD) are more sensitive to the VBI than are controls. According to the authors and to Kuhn and Land's (2006) assumptions, these results are surprising, because individuals with ASD are generally less sensitive to social cues (Dalton et al., 2005; Pelphrey et al., 2002; Riby \& Hancock, 2008; Sasson et al., 2007). To explain their results, Kuhn et al. (2010) proposed the hypothesis that individuals with ASD are sensitive to the illusion because they have trouble rapidly allocating attention to both people and moving objects. Our results demonstrated that a large proportion of participants (71 \% in Exp. 1 and $87.5 \%$ in Exp. 2) were sensitive to the VBI without social cues from the magician. Thus, whereas Kuhn et al.'s (2010) hypothesis offers an interesting perspective on ASD, the role of social cues in the VBI is probably more nuanced. The mechanisms responsible for this effect are still poorly understood by psychologists, and this perhaps opens up some interesting avenues for understanding human perception.

A potential hypothesis for explaining the psychological mechanism of the VBI is that, when exposed to the unexpected event (the ball "magically" disappearing), the mind tries to reduce the dissonance between prior expectations (the ball will move up) and the unexpected visual event (the ball does not move up) using three potential mechanisms, leading to three different experiences of the VBI.

First, prior expectations (that the ball will move up) could be ignored, and visual feedback (that the ball is gone before leaving the magician's hand) can be prioritized. If this mechanism is activated, participants will not see the ball moving up, and they will find the most effective solution to explain this event: that the magician still has the ball in his hand or in his sleeve.

Second, prior expectations and perceptual feedback could be combined. The result of this combination would be the mental picture of a ball moving up and disappearing within the screen. Here, participants might be sensitive to the VBI, and they would suppose that the most effective solution to explain this "incredible" event (the ball "disappearing" in midair) is the existence of a video edit.

Finally, prior expectations could be prioritized and perceptual feedback ignored. If this mechanism is activated, participants may be most sensitive to the VBI and will assume that the ball moved up and off the screen. This mechanism may be the most effective one and the one most frequently used. Moreover, because we know that a moving object is usually less visible due to its speed, the "ball is gone" visual feedback might be interpreted as "the elevating ball trajectory is invisible due to its high speed," and this interpretation is congruent with the expectation that "the ball will move up." As in the "representational momentum" effect, the perceptual bias of the VBI seems to be anchored in expectations rather than in the direction of the real action (e.g., Hubbard \& Bharucha, 1988; Hubbard \& Motes, 2005).

Finally, according to our hypothesis, sensitivity to the VBI may be linked to several interindividual differences in the resolution of the dissonance between prior expectations and the real visual feedback of a "magic" event. The 
close examination of the reasons why some participants believe what they see, while others "see" what they believe, might lead to a better understanding of our perception and some of its limits.

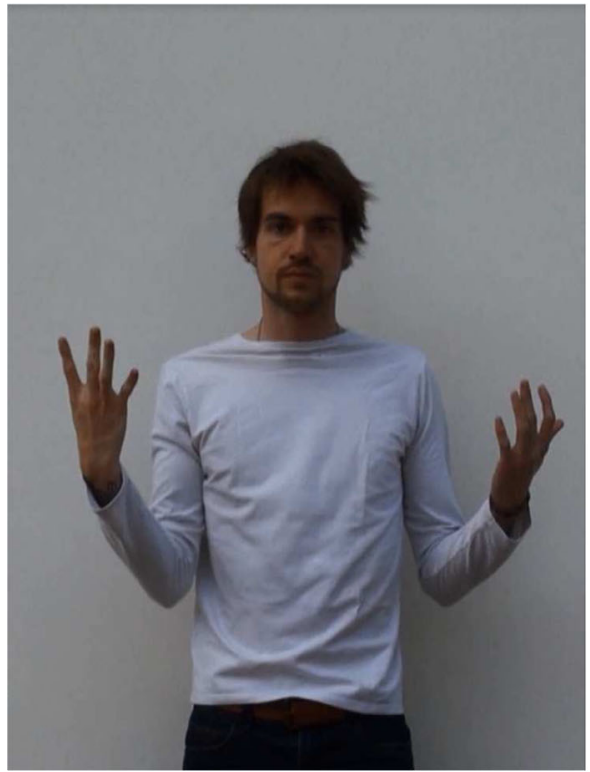

\section{Appendix A: Ball illusion questionnaire}

1. Point with the cursor to the location where you saw the ball for the last time on the computer screen. ${ }^{6}$

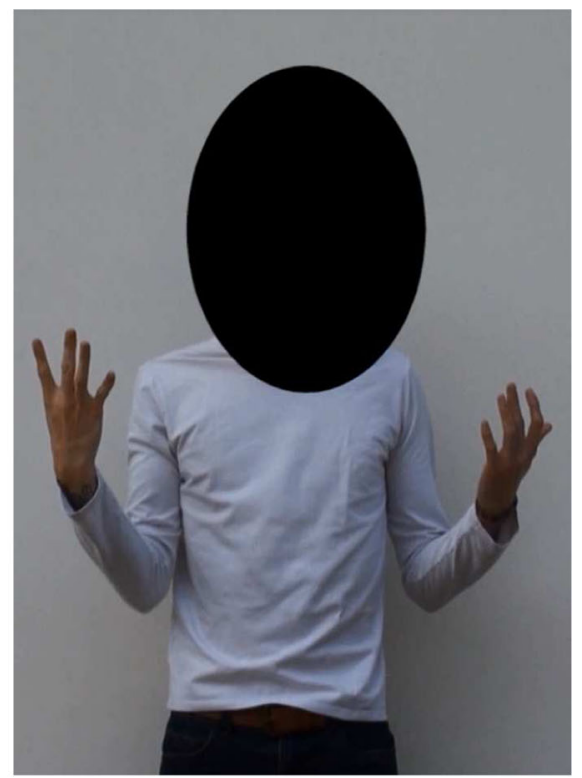

2. Describe what you saw:

3. How do you think the illusion was created?

4. Did you see the ball move up on the final throw? Yes or No

5. Did you see the ball move off the screen during the final throw? Yes or No

\section{Appendix B: Video links}

\section{Experiment 1}

Social-Cue Condition: https://www.youtube.com/watch?v= KMwZjKWUFNI

No-Social-Cue Condition: https://www.youtube.com/ watch? $\mathrm{v}=8 \mathrm{jGDqp}$ Vnlns

\section{Experiment 2}

Social-Cue Condition: https://www.youtube.com/watch?v= IVh9RZ cT9A

No-Social-Cue Condition: https://www.youtube.com/watch? $\mathrm{v}=\mathrm{TbYjMLDd} 8 \mathrm{Po}$

\section{Experiment 3}

Social-Cue Condition: https://www.youtube.com/watch?v= 61voX1NzWng

Anti-Illusion Condition: https://www.youtube.com/watch? $\mathrm{v}=$ SKTVBoj7TQ8

Fixed-Anti-Illusion Condition: https://www.youtube.com/ watch? $=$ PJ9wBmpYmPE

\section{References}

Cui, J., Otero-Millan, J., Macknik, S. L., King, M., \& MartinezConde, S. (2011). Social misdirection fails to enhance a magic illusion. Frontiers in Human Neuroscience, 5, 103. doi:10.3389/ fnhum.2011.00103

Dalton, K. M., Nacewicz, B. M., Johnstone, T., Schaefer, H. S., Gernsbacher, M. A., Goldsmith, H. H., ... Davidson, R. J. (2005). Gaze fixation and the neural circuitry of face processing in autism. Nature Neuroscience, $8,519-526$

Didierjean, A., Ferrari, V., \& Blättler, C. (2014). Role of knowledge in motion extrapolation: The relevance of an approach contrasting experts and novices. In B. H. Ross (Ed.), The psychology of learning and motivation (Vol. 61, pp. 215-235). San Diego: Academic Press.

\footnotetext{
${ }^{6}$ The picture with the black mask was only used in the no-social-cue condition of Experiment 1.
} 
Driver, J., Davis, G., Kidd, P., Maxwell, E., Ricciardelli, P., \& BaronCohen, S. (1999). Gaze perception triggers reflexive visuospatial orienting. Visual Cognition, 6, 509-541.

Freyd, J. J., \& Finke, R. A. (1984). Representational momentum. Journal of Experimental Psychology: Learning, Memory, and Cognition, 10, 126-132. doi:10.1037/0278-7393.10.1.126

Hegdé, J., Fang, F., Murray, S. O., \& Kersten, D. (2008). Preferential responses to occluded objects in the human visual cortex. Journal of Vision, 8(4), 16.1-16. doi:10.1167/8.4.16

Howard, C. J., \& Holcombe, A. O. (2010). Unexpected changes in direction of motion attract attention. Attention, Perception, \& Psychophysics, 72, 2087-2095. doi:10.3758/BF03196685

Hubbard, T. L. (2005). Representational momentum and related displacements in spatial memory: A review of the findings. Psychonomic Bulletin \& Review, 12, 822-851. doi:10.3758/BF03196775

Hubbard, T. L. (2015). The varieties of momentum-like experience. Psychological Bulletin, 141, 1081-1119. doi:10.1037/bul0000016

Hubbard, T. L., \& Bharucha, J. J. (1988). Judged displacement in apparent vertical and horizontal motion. Perception \& Psychophysics, 44, 211-221. doi:10.3758/BF03206290

Hubbard, T. L., \& Motes, M. A. (2005). An effect of context on whether memory for initial position exhibits a Fröhlich effect or an onset repulsion effect. Quarterly Journal of Experimental Psychology, $58,961-979$.

Kanizsa, G., \& Gerbino, W. (1982). Amodal completion: Seeing or thinking? In J. Beck (Ed.), Organization and representation in perception (pp. 167-190). Hillsdale: Erlbaum.

Kuhn, G., Caffaratti, H. A., Teszka, R., \& Rensink, R. A. (2014). A psychologically-based taxonomy of misdirection. Frontiers in Psychology, 5, 1392. doi:10.3389/fpsyg.2014.01392

Kuhn, G., Kourkoulou, A., \& Leekam, S. R. (2010). How magic changes our expectations about autism. Psychological Science, 21, 14871493. doi: $10.1177 / 0956797610383435$

Kuhn, G., \& Land, M. F. (2006). There's more to magic than meets the eye. Current Biology, 16, R950-R951. doi:10.1016/j.cub.2006.10.012

Kuhn, G., \& Rensink, A. R. (2015). The vanishing ball illusion: A new perspective on the perception of dynamic events. Cognition (in press)

Kuhn, G., Tatler, B. W., \& Cole, G. G. (2009). You look where I look! Effect of gaze cues on overt and covert attention in misdirection. Visual Cognition, 17, 925-944. doi:10.1080/13506280902826775
Kuhn, G., Teszka, R., Tenaw, N., \& Kingstone, A. (2015). Don't be fooled! Attentional responses to social cues in a face-to-face and video magic trick reveals greater top-down control for overt than covert attention. Cognition, 146, 136-142. doi:10.1016/j.cognition. 2015.08.005

Mansfield, E., Farroni, T., \& Johnson, M. H. (2003). Does gaze perception facilitate overt orienting? Visual Cognition, 10, 7-14.

Michotte, A., Thines, G., \& Crabbe, G. (1991). Amodal completion of perceptual structures. In G. Thines, A. Costall, \& G. Butterworth (Eds.), Michotte's experimental phenomenology of perception (pp. 140-167). Hillsdale: Erlbaum (Original work published 1964).

Pelphrey, K. A., Sasson, N. J., Reznick, J. S., Paul, G., Goldman, B. D., \& Piven, J. (2002). Visual scanning of faces in autism. Journal of Autism and Developmental Disorders, 32, 249-261.

Rensink, R. A., \& Kuhn, G. (2015). The possibility of a science of magic. Frontiers in Psychology, 6, 1576. doi:10.3389/fpsyg.2015.01576

Riby, D. M., \& Hancock, P. J. (2008). Viewing it differently: Social scene perception in Williams syndrome and autism. Neuropsychologia, 46, 2855-2860.

Sasson, N., Tsuchiya, N., Hurley, R., Couture, S. M., Penn, D. L., Adolphs, R., \& Piven, J. (2007). Orienting to social stimuli differentiates social cognitive impairment in autism and schizophrenia. Neuropsychologia, 45, 2580-2588. doi:10.1016/j.neuropsychologia.2007.03.009

Tachibana, R. (2014). The effects of social misdirection on magic tricks: How deceived and undeceived groups differ. i-Perception, $5,143-146$.

Tatler, B. W., \& Kuhn, G. (2007). Don't look now: The magic of misdirection. In R. van Gompel, M. Fischer, W. Murray, \& R. L. Hill (Eds.), Eye movement research: Insights into mind and brain (pp. 697-714). Amsterdam: Elsevier.

Thomas, C., Didierjean, A., Maquestiaux, F., \& Gygax, P. (2015). Does magic offer a cryptozoology ground for psychology? Review of General Psychology, 19, 117-128.

Triplett, N. (1900). The psychology of conjuring deceptions. American Journal of Psychology, 11, 439-510.

Tse, P. U. (1999). Volume completion. Cognitive Psychology, 39, 37-68. doi:10.1006/cogp.1999.0715

van Lier, R., \& Wagemans, J. (1999). From images to objects: Global and local completions of self-occluded parts. Journal of Experimental Psychology: Human Perception and Performance, 25, 1721-1741. doi:10.1037/0096-1523.25.6.1721 